\title{
sRNAs as possible regulators of retrotransposon activity in Cryptococcus gattii VGII
}

\author{
Patrícia Aline Gröhs Ferrareze ${ }^{1}$, Rodrigo Silva Araujo Streit ${ }^{2}$, Francine Melise dos Santos ${ }^{1}$, Augusto Schrank ${ }^{1,2}$, \\ Livia Kmetzsch ${ }^{1,2}$, Marilene Henning Vainstein ${ }^{1,2}$ and Charley Christian Staats ${ }^{1,2^{*}}$
}

\begin{abstract}
Background: The absence of Argonaute genes in the fungal pathogen Cryptococcus gattii R265 and other VGII strains indicates that yeasts of this genotype cannot have a functional RNAi pathway, an evolutionarily conserved gene silencing mechanism performed by small RNAs. The success of the R265 strain as a pathogen that caused the Pacific Northwest and Vancouver Island outbreaks may imply that RNAi machinery loss could be beneficial under certain circumstances during evolution. As a result, a hypermutant phenotype would be created with high rates of genome retrotransposition, for instance. This study therefore aimed to evaluate in silicio the effect of retrotransposons and their control mechanisms by small RNAs on genomic stability and synteny loss of C. gattii R265 through retrotransposons sequence comparison and orthology analysis with other 16 C. gattii genomic sequences available.
\end{abstract}

Results: Retrotransposon mining identified a higher sequence count to VGl genotype compared to VGII, VGIII, and VGIV. However, despite the lower retrotransposon number, VGIl exhibited increased synteny loss and genome rearrangement events. RNA-Seq analysis indicated highly expressed retrotransposons as well as sRNA production.

Conclusions: Genome rearrangement and synteny loss may suggest a greater retrotransposon mobilization caused by RNAi pathway absence, but the effective presence of sRNAs that matches retrotransposon sequences means that an alternative retrotransposon silencing mechanism could be active in genomic integrity maintenance of C. gattii VGIl strains.

Keywords: Cryptococcus gattii, RNAi, Retrotransposons, Synteny

\section{Background}

Transposable elements (TE) are repeated DNA sequences that move and propagate in genome by "copyand-paste" (TE class I) and "cut-and-paste" (TE class II) mechanisms [1]. TE class I, or retrotransposons, replicate through an RNA intermediate. Autonomous sequences encode their own reverse transcriptase and are typically $5-10$ kilobases in length. There are two classes of retrotransposons, based on the presence of LTR (long terminal repeats) or its absence (non-LTR retrotransposons - LINE/SINE elements) [2]. At each

\footnotetext{
* Correspondence: staats@cbiot.ufrgs.br

${ }^{1}$ Programa de Pós-Graduação em Biologia Celular e Molecular, Centro de Biotecnologia, Universidade Federal do Rio Grande do Sul (UFRGS), 91501-970 Porto Alegre, RS, Brazil

${ }^{2}$ Departamento de Biologia Molecular e Biotecnologia, Instituto de Biociências, Universidade Federal do Rio Grande do Sul (UFRGS), Porto Alegre, RS, Brazil
}

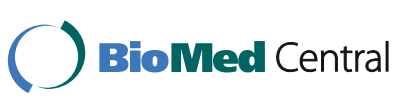

(c) The Author(s). 2017 Open Access This article is distributed under the terms of the Creative Commons Attribution 4.0 International License (http://creativecommons.org/licenses/by/4.0/), which permits unrestricted use, distribution, and reproduction in any medium, provided you give appropriate credit to the original author(s) and the source, provide a link to the Creative Commons license, and indicate if changes were made. The Creative Commons Public Domain Dedication waiver (http://creativecommons.org/publicdomain/zero/1.0/) applies to the data made available in this article, unless otherwise stated. end of LTR retrotransposons, there are repeats necessary for their replication cycle which contain sequences that regulate transcription. All LTR retrotransposons can have gag and pol genes; gag encodes proteins (GAGs; Groupspecific AntiGen) that form VLPs (Virus-Like Particles), and pol encodes a polypeptide composed of (i) integrase, (ii) ribonuclease $\mathrm{H}$, (iii) reverse transcriptase, and (iv) aspartyl protease enzymes [3]. Gag and pol genes are usually located in two open reading frames (ORFs), but in some LTR retrotransposons, a single gag/pol ORF is separated by a frameshift or a stop codon [4].

Transposable elements most commonly found in fungal species are retrotransposons. Some studies have elucidated significant effects of transposable elements as an evolutionary force, leading to synteny loss, avirulent gene deletion, and genome expansion $[1,5]$. Insertion of transposable elements near genes can lead to 
changes in gene expression patterns, while insertions within them can modify gene structure. Recombination between LTR elements in different sites leads to chromosomal rearrangements on a large scale. Because of their potentially deleterious effects, host organisms frequently evolve mechanisms to limit the activity of such elements [2].

The function of small RNAs as a defense mechanism against mobile elements is widely conserved among double-stranded RNA-mediated interference (RNAi) routes [6]. In recent years, several studies have examined the role of miRNAs in various cellular processes such as genomic integrity maintenance, DNA damage responses to biotic stress, and morphological process regulation. Interfering RNAs play critical roles in gene regulation, chromosomal structure, and genomic stability, such that the RNAi conserved function is to protect the genome from mobile element invasion [6]. The RNAi pathway mediates homology-dependent degradation of an mRNA with small RNA molecules and is a key regulatory mechanism that controls transcription and translation in eukaryotic organisms. The enzymes required for RNA silencing are numerous and may vary among species, but universally include Argonaute proteins that bind small RNAs, Dicer ribonuclease that produces small interfering RNAs (siRNA) from double stranded RNA precursors (dsRNA), and RNA-dependent RNA polymerase that produces dsRNAs [7]. In this process, a double stranded RNA produced by an RNA-dependent RNA polymerase is first processed into small interfering RNAs of 21-28 nucleotides by Dicer RNase III and enters the canonical pathway. This siRNA is incorporated into a nuclease complex, the RNA-induced silencing complex (RISC), containing an Argonaut protein with RNA-degrading activity. After the RISC complex, siRNAs promote the cleavage of homologous mRNAs [8]. These factors play a key role in eukaryote genome defense: null mutations in their corresponding genes result in increased transposons expression and mobilization, decrease and loss of endogenous retrotransposon-derived siRNAs, and drug resistance mutations induced by retrotransposons [9-11].

Two species from the genus Cryptococcus are the etiologic agent of cryptococcosis. Cryptococcus neoformans has worldwide distribution and affects immunocompromised individuals, mainly causing cryptococcal meningoencephalitis. Cryptococcus gattii, prior to the Pacific Northwest outbreak, was known to be endemic in tropical and subtropical areas, typically infecting healthy hosts with pulmonary cryptococcosis $[12,13]$. Estimated numbers calculate that $C$. neoformans cryptococcosis kills 650,000 immunocompromised HIV/AIDS patients every year worldwide [13]. However, the common clinical indistinction between $C$. neoformans and C. gattii during cryptococcosis diagnosis can underestimate the real number of cases associated with C. gattii cryptococcosis. Studies in a murine model of pulmonary cryptococcosis have demonstrated that there is no significant difference in mortality rates of mice infected with $C$. neoformans H99 and the hypervirulent strain C. gattii R265 [14].

The $C$. neoformans serotype $\mathrm{D}$ contains two paralogous genes for Argonaute (AGO1 and AGO2) and Dicer (DCR1 and DCR2), and one gene for RdRP (RDP1). In contrast, serotype A has a single Argonaut gene (AGO1) and RdRP (RDP1) and two paralogous genes for Dicer (DCR1 and DCR2). C. gattii has four molecular types (VG "variety gattii"): VGI and VGII are the main pathogens of cryptococcosis in immunocompetent hosts, while VGIII and VGIV rarely cause infections and affect immunocompromised hosts [15]. VGI, VGIII, and VGIV genotypes have all the components of an RNAi system, while VGII has a canonical DCR2 and DCR1/RDP1 pseudogenes [16]. Bielska and May [13] have shown that $97 \%$ of documented cases of cryptococcosis in the Vancouver Island outbreak were caused by VGII, with VGIIa prevalent in more than $80 \%$ of the cases.

The absence of Argonaute genes in the primary pathogen C. gattii R265, and other VGII strains, indicates that these genotypes do not have a functional RNAi pathway [16] . The success of the R265 strain as a pathogen, in causing the Vancouver Island and Pacific Northwest outbreaks, may imply that RNAi machinery loss can be beneficial under certain circumstances during evolution [6]. This can be linked to the higher recombination rates of VGII strains, which are associated with the generation of novel and possibly more virulent genotypes [17]. The loss of RNAi could thus allow greater mobility of transposable elements, as has been reported in C. neoformans [18]. However, in the RNAi-lacking budding yeast Saccharomyces cerevisiae, a predominant proportion of sequenced small RNAs mapped to transposable elements [5]. Recent analysis showed that some yeasts, which have presumably lost the RNAi route, actually have siRNAs and use non-canonical Dicer proteins to control Argonaute-like proteins without RdRP [8]. Therefore, an alternative retrotransposon silencing mechanism by sRNAs may act through a non-canonical RNAi pathway or through independent control systems.

Thus, the study of retrotransposon effects in C. gattii genomes, as a hypervirulent strain, with potentially high activity of transposable elements unrelated to any silencing mechanism, may contribute to increased understanding of the role of retrotransposons and sRNAs in genome evolution.

\section{Results}

Retrotransposon mining in C. gattii genome sequences

Retrotransposon structure usually consists of two almost identical LTRs flanking two ORFs to the gag and pol 
genes [19]. Retrotransposon mining programs rely on the search for several structural features of LTR retrotransposons, such as the size range of the LTR sequences, the distance between the two LTRs of an element, among others [20]. The number of LTRretrotransposons identified in 17 C. gattii genomes ranged from 21 to 78 sequences (Fig. 1). Their structural composition was confirmed using NCBI Blast (Conserved Domains) for the presence of gag and pol domains (Additional file 1). In this search, non-autonomous LTR sequences (which do not encode necessary proteins for transposition) were excluded. Reciprocal BLASTn (data not shown) and domain arquitecture present in the predicted proteins (Additional file 1) revealed that such sequences groups are unique. As shown in Fig. 1, VGI strains (WM276, NT10, E566, EJB2, and RU294), which putatively contain a complete RNAi pathway, present a higher retrotransposon count (especially WM276 and NT10) when compared to VGII (R265, CA1014, CBS7750, CBS10090, RAM5, LA55, MMRL2647, 99473, and 2001935), VGIII (CA1280 and CA1873), and VGIV (IND107) strains.

\section{Retrotransposon orthology and synteny evaluation}

Retrotransposon integration is a non-random process in the genome, as many transposable elements integrate into specific sequences as intergenic regions or upstream of RNA polymerase III-transcribed genes [21]. Copia elements (Ty1 family: Pseudoviridae) present specificity for pre-existing LTR transposons (resulting in a TE cluster formation) and transcriptionally active regions, while gypsy elements (Ty3 family: Metaviridae) are reported in heterochromatin [22]. Preferential insertion of TE clusters in regions with low recombination rates can be explained by weak selection against these sites [21]. For these reasons, most filamentous fungi carry more Ty3/ gypsy than Ty1/copia elements [22]. The presence of retrotransposon-enriched regions (TE clusters) can be observed by sequence distribution along the 14 chromosomes of WM276 (Additional file 1). However, the 73 retrotransposon elements detected in the NT10 genome are generally incomplete (absence of conserved domain) and dispersed along the genome. A slight prevalence of Ty3/gypsy was found in the element composition of $C$. gattii strains.

The WM276 strain has 78 retrotransposon sequences, of which 24 are unique and 29 shared only with VGI strains. The R265 strain has 34 sequences, five of which are unique with 14 exclusive to VGII strains. Unique sequences indicate recent transposition events, since they do not have identifiable orthologues. Figure 2 shows the relationship between sequence amount and orthologue number. VGII strains (mainly CA1014) share more sequences with R265 than other genotypes. Similarly, WM276 shares 49 sequences with VGI strains. These results indicate the existence of transposition events after genotype diversification. Data analysis has allowed the observation of the prevalence (almost exclusivity) of $g a g$ domains in Ty1 family sequences in the absence of aspartic protease. However, Ty3 has aspartic protease domains and is lacking gag. The presence of RVT_1 (Pfam: PF00078) and RVT_2 (Pfam: PF07727) reverse transcriptase families have been observed in gypsy and copia, respectively.

The chromodomains identified next to retrotransposons WM276.1, 53, 72, and 78 can be related to the

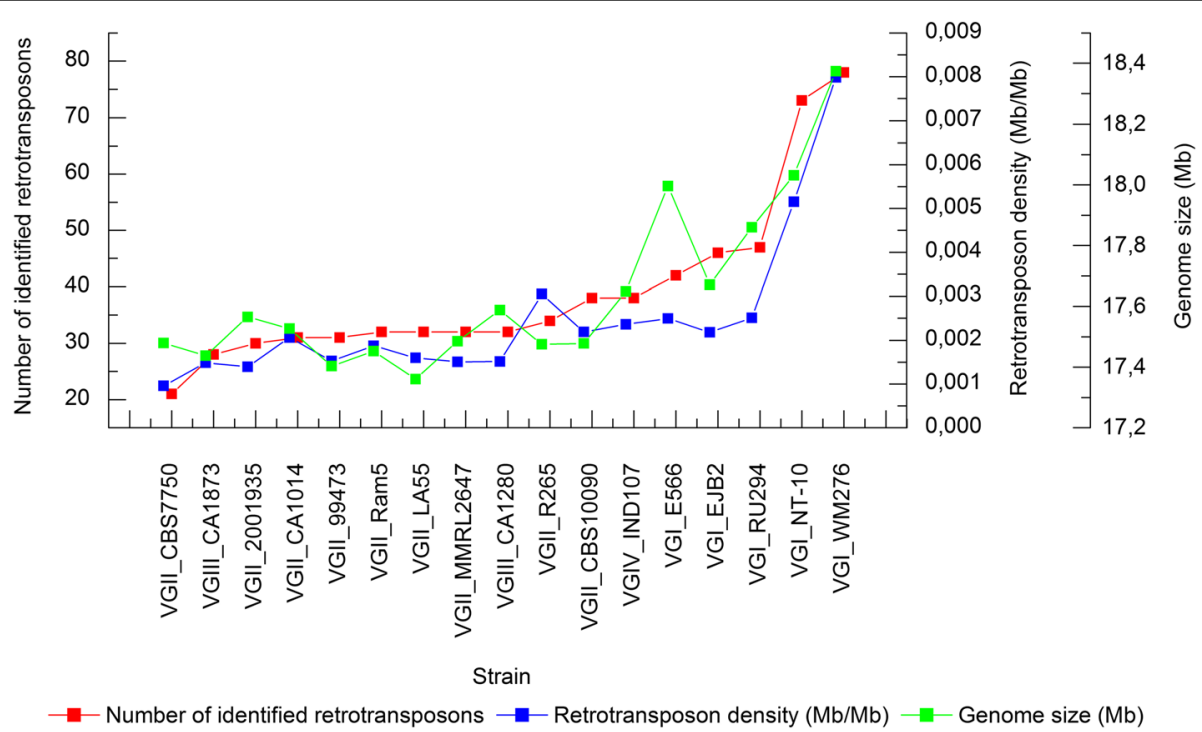

Fig. 1 Retrotransposon number, density and genome size comparison. The red line indicates the number of identified retrotransposons in each strain; blue line represents retrotransposon density in C. gattii genomes. The green line shows the size of C. gattii assembled genomes 


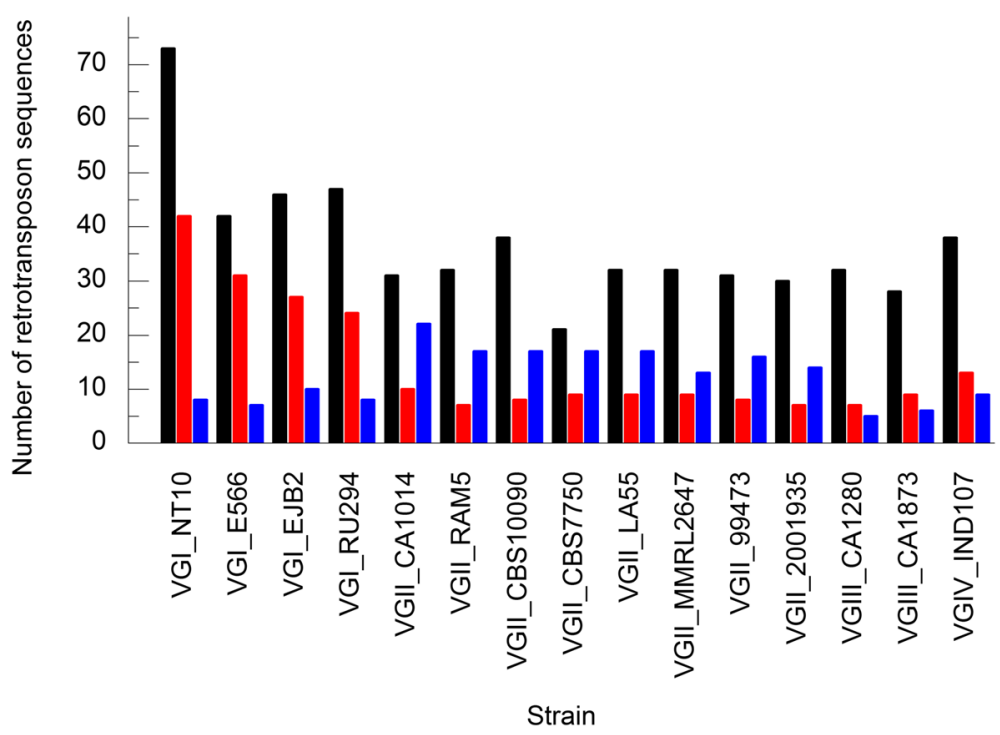

Number of identified retrotransposons

Retrotransposons with orthologous sequences in WM276 strain

Retrotransposons with orthologous sequences in R265 strain

Fig. 2 Orthologous sequences count. Orthologous sequences from C. gattii WM276 and R265 shared with VGI, VGII, VGIII, and VGIV strains

Chromovirus genus of the Metaviridae (Ty3) family. These chromodomains, absent in R265 strain, are found in functional proteins as well as gypsy LTR retrotransposons. The canonical function includes chromatin remodeling and gene expression regulation. However, chromodomains in retrotransposons are poorly understood [23].

Genome rearrangements naturally accumulate in different strains. Successive rearrangements in C. gattii and C. neoformans genomes may have contributed to sexual isolation and speciation [24]. To determine the effect of retrotransposon mobilization in VGI and VGII strains, their synteny conservation was evaluated (Additional file 1). Orthologous elements shared by VGI and VGII genotypes were analyzed for synteny on a multiple genome alignment. Multiple alignments were performed using the Mauve program with WM276 as a reference for supercontig reordering. VGI strain alignment identified 29 synteny blocks in WM276 (Additional file 2) while VGII alignment found 54 synteny blocks in R265 (Additional file 3), indicating greater genome fragmentation or a better VGI genome sequence assembly. Both alignments had few rearrangements, with a predominant collinear genome. Coordinates of orthologous retrotransposons were used to classify syntenic and non-syntenic sequences.

Long terminal repeats from LTR retrotransposons are known to promote crossing over at non-homologous sites, leading to chromosomal rearrangement. Likewise, mutations arising from mobile element insertions in conserved blocks can cause synteny loss [21]. TE clusters from WM276 were shown to be responsible for synteny breaks at 5 genome points (Additional files 4, 5, 6,7 and 8). The same number was found in R265 (Additional files 9, 10, 11, 12 and 13), suggesting that R265 active retrotransposons, even if in a small identifiable number, are more influential on genome integrity than WM276 sequences.

C. gattii R265 strain retrotransposons have 133 orthologous sequences in 8 VGII genomes. The majority of these $(65.41 \%)$ preserve synteny with the R265 genome (Fig. 3a), particularly the CBS7750 strain, which has 17 orthologous sequences in syntenic blocks with R265. In comparison, VGI, despite its higher elements count, has almost $80 \%$ of orthologous sequences in synteny with the WM276 genome (Fig. 3b).

Among the widely distributed orthologous sequences of R265.3, R265.19, and R265.22 retrotransposons, the multiple genome alignment of 17 C. gattii strains shows synteny conservation for most of the C. gattii strains (Additional file 14). However, some variations suggest genome rearrangement events (Additional file 15). Orthologs of retrotransposon R265.3 identified in RU294 (VGI), IND107 (VGIV), and CA1280 (VGIII) are localized in the same block and generate their own syntenic group, while orthologs of retrotransposon R265.19 are kept syntenic in all genomes of these three genotypes. Among VGII, orthologs of retrotransposon R265.3 and orthologs of retrotransposon R265.19 sequences were located in opposite positions, such as an ectopic recombination result. This sequence inversion was found in CA1014, CBS10090, MMRL2647, and 2001935. Moreover, CA1014 and CBS10090 each present an exclusive 


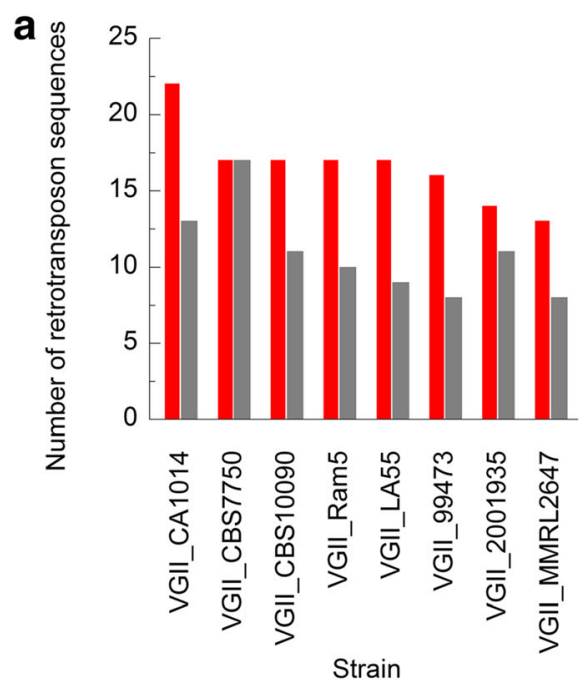

Retrotransposons with orthologous in R265 strain

Retrotransposons that are synthenic with R265 orthologues

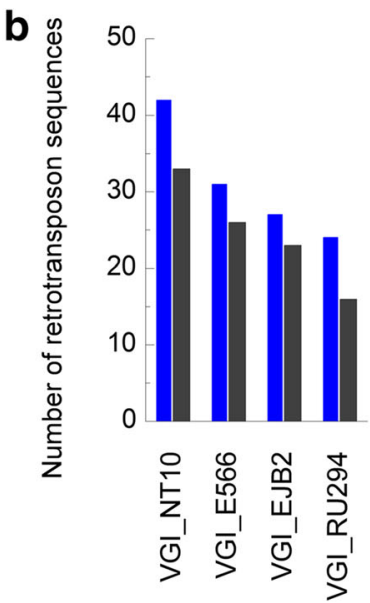

Strain

Retrotransposons with orthologous in WM276 strain

Retrotransposons that are synthenic with WM276 orthologues

Fig. 3 Sequence count of orthologous and syntenic retrotransposons. Bars indicate the total amount of orthologous and syntenic sequences shared by VGII (a) and VGI (b) strains with R265 and WM276 genomes, respectively

syntenic location to sequence orthologs of retrotransposon R265.22.

Synteny conservation at retrotransposon orthologous sites indicates low mobilization rates and reveals that an RNAi pathway effectively acts in WM276 to protect the genome against retrotransposon propagation. In this context, it is feasible to assume that there are additional mechanisms protecting the VGII strain genomes from mobile elements.

\section{Phylogenetic inference}

Among the 34 retrotransposons belonging to the R265 genome, three RT sequences (named R265.3, R265.19, and R265.22) have orthologues present in all 17 C. gattii strains. Of these, only one (R265.19) was retained in $C$. neoformans H99. The RT R265.19 has two conserved domains (reverse transcriptase and RNase $\mathrm{H}$ ) in all orthologous sequences (except in H99, where the sequence is inserted in a TE cluster) and was used as a model for the phylogenetic evaluation of $C$. gattii retrotransposon propagation. The evolutionary model selected for nucleotide substitution was Kimura 2-parameters [25]. The phylogram (Fig. 4) shows C. gattii diversification through the evolutionary path taken by reverse transcriptase (Fig. 4a) and RNase H (Fig. 4b) sequences. In both cases, monophyletic clades for 4 C. gattii genotypes are identifiable. Although the reverse transcriptase domain seems to have undergone more modifications, both trees indicate the relationship between VGI, VGIII, and VGIV, as well as the basal separation of the VGII clade. Early divergence and isolation of VGII molecular types as well as the late separation of VGI, VGIII, and VGIV from a common ancestor has been proposed by Farrer and colleagues [15] in an evolutionary relationship reconstruction for $C$. gattii genotypes.

\section{Expression evaluation}

The activation of transposable elements in response to stress conditions usually involves regulatory sequences at promoter regions, which are similar to motifs required for the induction of stress-responsive genes [26] as well as the preferential integration of some type of retrotransposon sequence into the promoters of stress response genes [27]. Different stress conditions, such as ionizing radiation, reactive oxygen species (ROS), DNA damage, and nutrient starvation can activate transcription and retrotransposition [26, 28]. Hence, element mobilization provide a means for the rapid evolution of gene regulatory networks, mediating the adaptive response to stress conditions [27]. Therefore, we speculated that stress conditions caused by zinc starvation in wild-type (WT) cells and cells lacking the ZAP1 gene mutant would alter retrotransposon expression. Zinc restriction was show to induces oxidative stress in yeast cells [29-31]. ZAP1 codes for a master zinc regulator in C. gattii and its is characterized in some fungal species [32]. Its absence was show to lead to reduction of anti- 

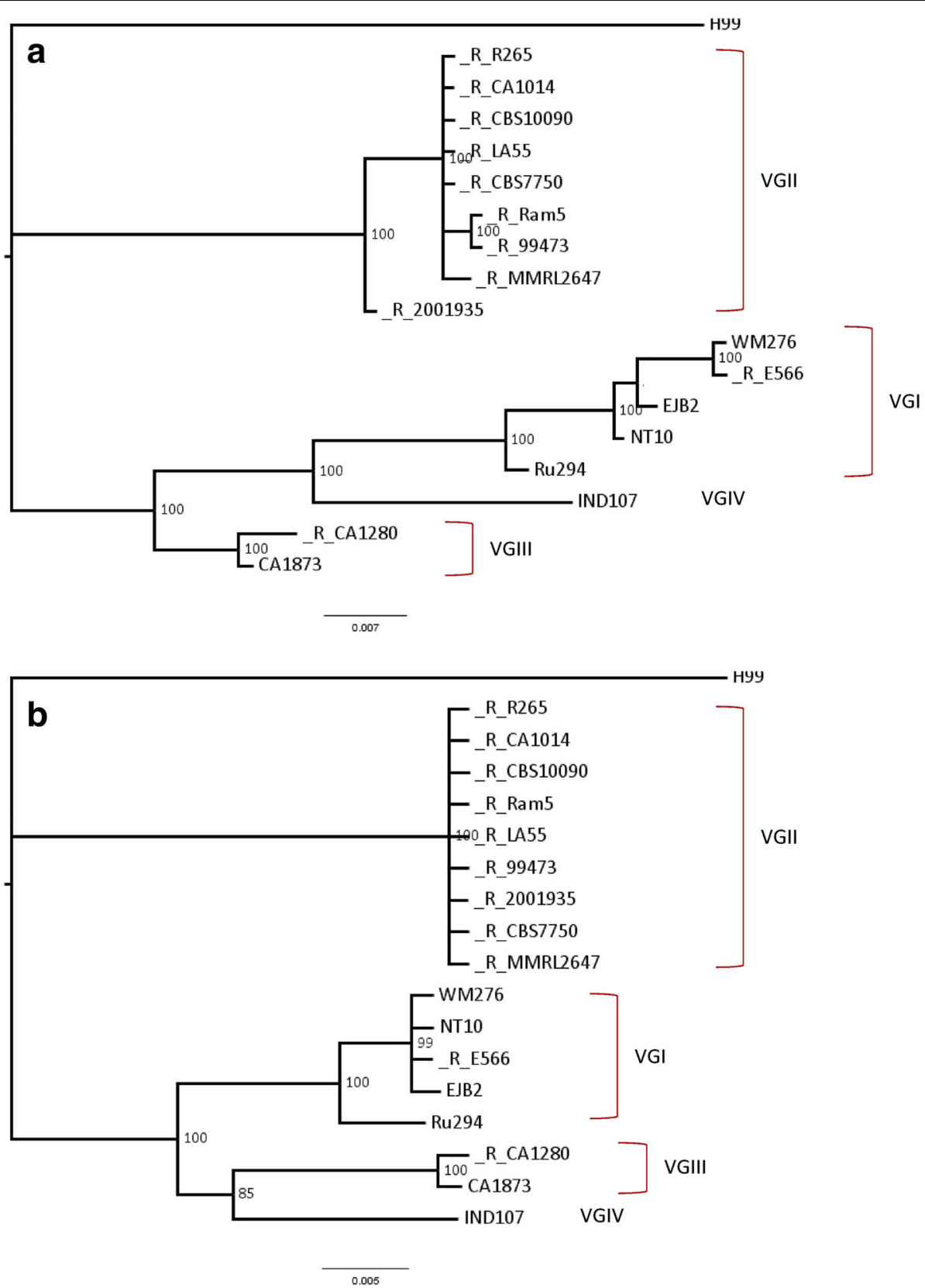

Fig. 4 Phylogenetic tree for reverse transcriptase (RT) and RNase H domains. Domains of reverse transcriptase (a) and ribonuclease $\mathrm{H}$ (b) from C. gattii retrotransposons to orthologous R265.19 sequence. Sequences that were direction-adjusted by MAFFT aligner are indicated by the "R" before strain name. Model inference was performed by jModelTest and phylogeny reconstruction by Mr. Bayes using the Kimura-2-parameters substitution model

oxidative responses in $C$. gattii cells [33] as well in $S$. cerevisiae $[29,34]$.

RNA-Seq data from C. gattii R265 WT and a zap1 mutant grown in YNB media with zinc deprivation were used to evaluate retrotransposon expression and silencing in stress conditions. The read counts of aligned libraries were quantified and the $\log 2$ fold change of differential expression analysis was quantified and normalized to Reads Per Kilobase of tanscript per Million mapped reads (RPKM) values for transcription measurement (Table 1, Additional file 16). No drastic changes in the RPKM values or the number of mapped reads distribution could be detected for both conditions (Additional files 17 and 18). Despite the variability of expression among different retrotransposons, no differential expression of such elements between WT and zap1 strains could be detected. The only two exceptions were RTs R265.26 and R265.38, which are only detected in WT or 
Table 1 RPKM and log2 fold change values in retrotransposon sequences

\begin{tabular}{|c|c|c|c|c|}
\hline Retro transposon & RPKM sRNA_WT & RPKM mRNA_WT & RPKM mRNA_ZAP1 & Log2 fold change (mRNA) ZAP1xWT \\
\hline R265.1 & 2212.17 & 165.12 & 48.09 & -1.78 \\
\hline R265.10 & 2728.76 & 182.41 & 318.81 & 0.80 \\
\hline R265.11 & 8396.39 & 14638.41 & 15671.02 & 0.10 \\
\hline R265.12 & 13322.54 & 133.26 & 16.63 & -3.00 \\
\hline R265.13 & 3991.29 & 2050.13 & 677.89 & -1.60 \\
\hline R265.14 & 8884.66 & 80.78 & 70.59 & -0.19 \\
\hline R265.15 & 1676.88 & 0 & 0 & N/A \\
\hline R265.16 & 19299.97 & 6745.21 & 11206.42 & 0.73 \\
\hline R265.17 & 808.77 & 0 & 27 & N/A \\
\hline R265.18 & 5015.68 & 107.47 & 125.22 & 0.22 \\
\hline R265.19 & 48521.82 & 29562.31 & 36487.05 & 0.30 \\
\hline R265.2 & 2923.34 & 360.06 & 125.86 & -1.52 \\
\hline R265.20 & 2567.82 & 168.44 & 183.99 & 0.13 \\
\hline R265.21 & 4481.6 & 23880.77 & 25869.49 & 0.11 \\
\hline R265.22 & 45424.93 & 259.33 & 226.62 & -0.19 \\
\hline R265.23 & 3496.62 & 28.71 & 25.09 & -0.19 \\
\hline R265.24 & 1074.35 & 145.76 & 42.46 & -1.78 \\
\hline R265.25 & 18917.41 & 9940.13 & 12131.12 & 0.29 \\
\hline R265.26 & 2075.06 & 511.17 & 0 & N/A \\
\hline R265.27 & 4526.67 & 103.71 & 30.21 & -1.78 \\
\hline R265.28 & 1553.01 & 40.86 & 35.71 & -0.19 \\
\hline R265.29 & 5431.2 & 496.81 & 186.06 & -1.42 \\
\hline R265.3 & 19795.43 & 7875.53 & 9215.24 & 0.23 \\
\hline R265.30 & 9472.7 & 2621.35 & 52.66 & -5.64 \\
\hline R265.31 & 5159.63 & 129824.4 & 90122.72 & -0.53 \\
\hline R265.34 & 4506.72 & 0 & 0 & N/A \\
\hline R265.36 & 103818.7 & 10138.81 & 20509.48 & 1.02 \\
\hline R265.37 & 39301.07 & 5627.55 & 22949.74 & 2.03 \\
\hline R265.38 & 3792.86 & 0 & 35.2714 & N/A \\
\hline R265.4 & 4525.26 & 75.71 & 132.32 & 0.80 \\
\hline R265.5 & 24813.18 & 22065.62 & 31063.56 & 0.49 \\
\hline R265.6 & 37947.45 & 514.37 & 345.77 & -0.57 \\
\hline R265.7 & 22013.22 & 17762.5 & 16862.24 & -0.07 \\
\hline R265.8 & 3893.5 & 241.84 & 1704.8 & 2.82 \\
\hline
\end{tabular}

zap1 conditions, respectively. This suggests that the absence of Zap1 transcription factor in a zinc-limiting environment alters the activity of such retrotransposons. Thus, it is possible that motif recognition on the retrotransposon promoter or a genomic localization near to Zap-1 target genes may be the cause of expression decline.

Retrotransposon propagation leads to genomic instability, and as a consequence, genetic diversity and phenotypic variation [35]. However, to avoid deleterious effects, there are several multiple transcriptional and posttranscriptional repressing mechanisms [36]. Among these mechanisms are those mediated by small RNAs, as previously described for the RNAi proficient $C$. neoformans H99 strain [9]. We therefore speculated that sRNAs could be controlling the expression of retrotransposons in C. gattii R265. We determined the sequence of small RNAs recovered form C. gattii R265 grow in zinc limitation conditions. Read length analysis revealed an abundance pattern somewhat similar to those observed in RNAi-proficient C. neoformans H99. While $C$. gattii most abundant reads ranges from 14 to 26 nt (data 
not shown), the majority of reads form $C$. neoformans library ranges from 21 to $23 \mathrm{nt}$ [9]. The presence of high mRNA expression with low sRNA associated levels (R265.31, R265.21) and high sRNAs levels with low expressions of mRNAs (R265.36, R265.22, R265.37, R265.6) may indicate an sRNA-regulated silencing mechanism for retrotransposon propagation. The correlation of mRNA $x$ sRNA RPKM values from WT libraries is exhibited in Fig. 5.

The mapping profile of sRNAs and mRNAs from the WT condition in the two most highly expressed fulllength retrotransposons (R265.5 and R265.7) can be observed in Fig. 6. Domain loss in many sequences, as well as the mutations and frameshifts in coding regions that render several domains non-functional, hampers the correct evaluation of domain expression patterns for all retrotransposons. Visually, sRNAs seem to be widely distributed and mRNAs are more abundant in LTR-5' (Additional files 19, 20 and 21). Statistically, the comparison between read counts of mRNAs distributed in LTRs, protein domains, and spacer regions (SR) exhibits significant a difference $(p<0,0001$ to $p<0,05)$ due to the increased values of LTR- $5^{\prime}$ read counts compared to other regions. As previously noted, long terminal repeats (LTRs) are important for transcription regulation (LTR-5', specifically, contains the promoter sequence) and usually contain high amounts of aligned reads. The absence of statistical variation inside the internal region of retrotransposons (consistent with the entire retrotransposon transcription) and the higher density of RNA-Seq reads mapping to LTR-5' was

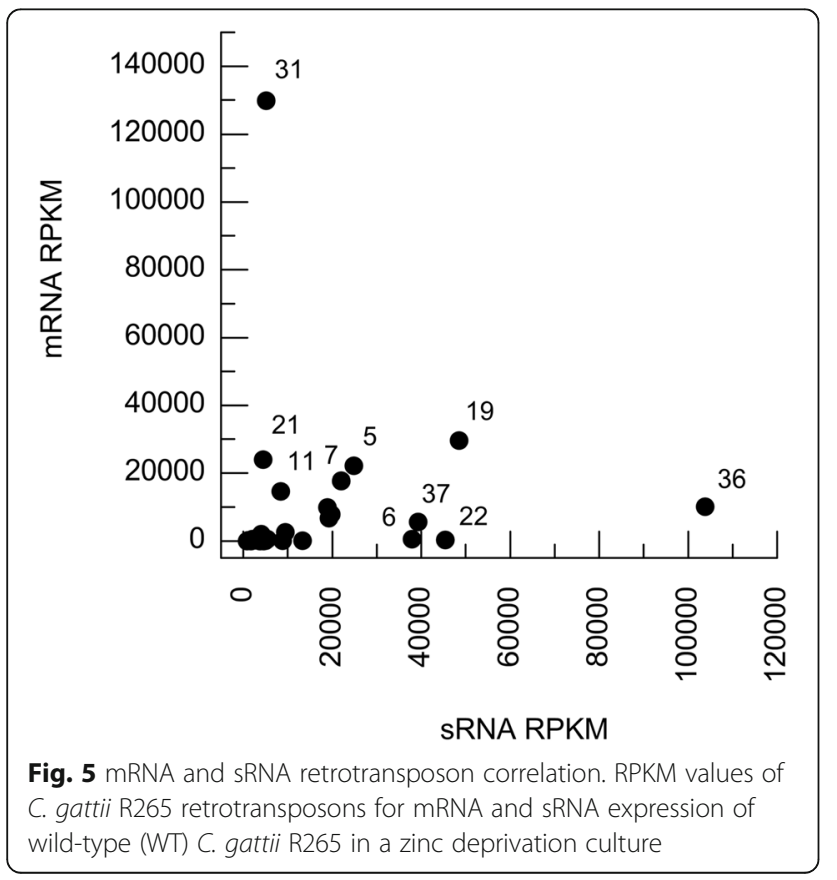

previously observed [37] in a large-scale LTR retrotransposon analysis of transcriptome data.

The sRNA profile analysis of raw read counts with R265.5 and R265.7 sequences reveals a decrease in aligned reads to protein domain sequences, especially reverse transcriptase (RT) and protease (PROT). As reported by Domingues and colleagues [38], most LTR retrotransposon families do not have high sRNA counts within the coding domains. The increased sRNAs read count in both LTRs (LTR-5' with statistical significance) and spacer regions (SR1, SR2, and SR3) shows the possible target regions for sRNA activity in a silencing mechanism.

\section{Discussion}

Retrotransposons are ubiquitously distributed in fungal species, albeit their structure is not conserved. The pol gene encodes a polyprotein composed of an aspartic protease, a reverse transcriptase, a ribonuclease $\mathrm{H}$, and an integrase; the order and number of domains may differ between retrotransposon families, and most of the elements have incomplete pol genes. In addition, retrotransposons are generally characterized by the presence of LTR [22]. As previously described [2], C. gattii retrotransposon sequences can be grouped into (i) LTRs with associated, but incomplete, internal regions; and (ii) partial internal regions with no identified LTRs.

The comparative analysis of $C$. gattii retrotransposons showed here revealed a large diversity of retrotransposons among diverse genotypes. It is noteworthy that the number of retrotransposons families number changes in a genotype-associated fashion. The VGI genotype strains possess the higher detectable retrotransposons and consequently the higher density compared to strains from VGII, VGIII and VGIV genotypes. This diversification of the number of retrotransposon families among different fungal strains is in agreement with previous reports comparing the prevalence of such mobile elements among closely related species and strains of Coccidioides immitis and Coccidioides posadasii [39], as well for Paracoccidioides spp. [40].

The genomic sequence of 16 C. gattii strains are available and their analysis described. Comparative genomic analysis revealed that chromosomal structure was very conserved among the four varieties (VGI, VGII, VGIII, and VGIV). Furthermore, this conservation is even more pronounced among VGII strains [15]. As expected, our retrotransposon conservation analysis revealed the majority of these mobile elements present in R265 strain have orthologs in other C. gattii strains, most of them displaying also synteny.

Strains of the VGII genotype do not have a functional RNAi pathway, a highly conserved mechanism to control and inhibit retrotransposon expression through a 

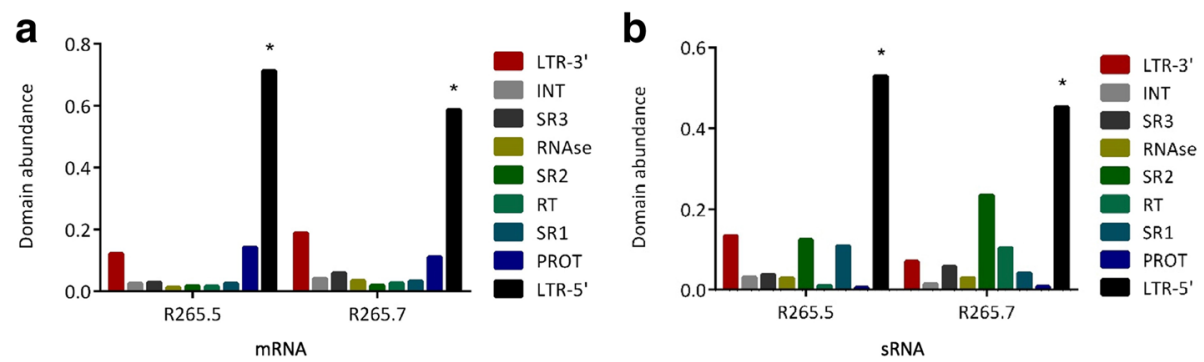

Fig. 6 mRNA and sRNA reads profile mapping. Distribution of aligned reads from a mRNA and $\mathbf{b}$ sRNA libraries in terminal repeats, protein domains, and spacer regions inside retrotransposon sequences. Abbreviations: Long Terminal Repeats (LTR), Integrase (INT), Ribonuclease H (RNAse), Reverse Transcriptase (RT), Aspartyl Protease (PROT)

homology-dependent gene-silencing complex [16]. It is therefore feasible to assume that VGII strains do not have an active RNAi pathway and that increased retrotransposons numbers are a consequence of increased retrotransposon expression and mobility, as seen in RNAi-deficient mutant of C. neoformans [8]. Unexpectedly, VGII strains displayed a smaller number of retrotransposon sequences compared to RNAi-proficient cryptococcal genotypes. Although R265 hypervirulence seems related to the absence of this regulatory mechanism, this observation suggests the existence of alternative control pathways or non-canonical Argonaute proteins, or the possibility that C. gattii (and not only VGII) has a more efficient (RNAi independent) system to control mobile element propagation. A predominance of LTR and DNA elements, LINEs, and lowcomplexity repeats in certain VGI strains was observed in C. gattii genotype analysis [15]. Furthermore, AGO gene absence in R265 and other VGII strains was not associated with gaps in the R265 genome [24]. However, it is unclear how far the incomplete assembly of genomes may alter the retrotransposon count. WM276 and NT10 have more retrotransposons and a wellassembled genome (WM276). Nevertheless, EJB2, E566, RU294, as both VGIII and VGIV strains, have RNAi pathways and exhibit similar sequence counts. The same explanation was suggested by Muszewska and colleagues [22] when analyzing differences in retrotransposon amounts between distinct strains of two Coccidioides species. Their findings further support that identified element amounts correlate with genomic sequences assembly lengths.

The retrotransposon expression analysis showed here revealed that all retrotransposons have detectable transcripts in distinct stress conditions. An uneven distribution of reads was detected among the C. gattii R265 transposons, with the large majority of reads matching one of the LTRs. This is in accordance to RNA-Seq assays using mRNA isolated from fission yeast [37].
To further evaluate retrotransposons expression, we performed a comparative transcriptome analysis using RNA-Seq data from C. gattii WT and zap1 mutant strains cultured under zinc restriction [33]. Iron, copper, and zinc are essential metals for many processes in fungal pathogens. Zinc, specifically, is involved in transcriptional control, ROS detoxification, carbohydrate oxidation, and alcoholic fermentation [41, 42]. Thereby, in Cryptococcus, the ZIP family of plasma membrane transporters mediates zinc acquisition, especially in zinc-limiting conditions. The expression regulation of these transporter-encoding genes is performed by the transcriptional factor $Z A P 1$. Our group previously demonstrated a severe impairment in ROS detoxification in cryptococcal cells lacking $Z A P 1$ gene [33]. This can be explained by the absence of indirect activation of many biological processes affected by zinc depletion in the zap1 mutant, since the complete removal of this metal harms all cellular functions of zinc-dependent metalloproteins. In this way, the comparison of WT and zap1 mutant cells allowed us to explore two distinct stress related conditions. However, our comparative analysis allowed the identification of only 2 stress-regulated retrotransposons.

Control of retrotransposon expression in fungal species is poorly characterized. Heterochromatin formation by $\mathrm{H} 3 \mathrm{mK} 9$ histone methyltransferase recruitment in Schizosaccharomyces pombe is mediated by specific siRNAs acting in an Argonaute complex [43, 44]. Saccharomyces cerevisiae (which lacks the RNAi machinery for silencing mobile elements) produces antisense non-coding RNAs to regulate gene expression at the transcriptional level [26] and post-transcriptionally, as an auxiliary in copy number control mechanism. This maintains lower levels of mature integrase and reverse transcriptase, coupled with a truncated gag [45]. At the transcriptional level, Ty1 antisense RNAs regulate Ty1 retrotransposon expression by alterations in chromatin function [45]. 
In C. neoformans, retrotransposon activity is controlled by the RNAi pathway [8]. Stalled spliceosomes associate with a complex named SCANR, leading to the formation of siRNAs responsible for retrotransposon silencing via AGO dependent RNA degradation [9]. As C. gattii VGII strains lack components of RNAi pathway, it is reasonable to assume that this strategy is not used by VGII strains to control retrotransposons activity. However, the detection of sRNAs that map to retrotransposon sequences with low expression claims that additional mechanisms for retrotransposon activity control may exist in C. gattii VGII strains. It is noteworthy that $C$. gattii R265 possess a functional DCR2 gene, which codes the enzyme responsible for the processing of siRNA precursors. In C. neoformans, Dcr2 was shown to be important for RNAi-mediated gene silencing [8]. In addition, the $C$. gattii VGII genomes accumulate a large amount of lost genes [16] and a hypervirulent phenotype. The absence of a canonical RNAi pathway can be a major influence on this profile. Nevertheless, the production of sRNAs means that C. gattii R265 has a secondary, alternative retrotransposon silencing mechanism, which guarantees genetic variation without the deleterious invasion of parasitic elements.

\section{Conclusions}

WM276 (VGI) is the only strain with a well-assembled genome and is therefore used as a parameter for predicting results in C. gattii analyses. Differences in retrotransposon count can be explained by incomplete genome assemblies. This is mainly because VGIII and VGIV strains, which are genetically more similar to VGI, have a functional RNAi pathway, but also have low numbers of retrotransposons (as VGII) when compared to WM276 and NT10. Sequences grouped in TE clusters are a strong feature of WM276 retrotransposons. Retrotransposon orthology showed the prevalence of shared sequences with related strains, a result of "ancient" retrotransposons from before C. gattii diversification. The unique sequences of WM276, if not a genomic assembly consequence, may indicate recent transposition events. Synteny conservation reflects RNAi importance in the control of mobile element propagation. VGI molecular type has superior rates of synteny maintenance within genotypes than VGII, and the same number of retrotransposon-associated synteny block breakpoints. This confirms that R265 retrotransposons exert an effect on genome stability. Besides differences in TE content, the phylogenetic inference of the R265.19 element demonstrated that retrotransposon propagation by nucleotide substitution analysis resembles $C$. gattii strain expansion, also showing a greater conservation of RNase and reverse transcriptase in VGII. In addition, expression evaluation confirms the existence of retrotransposons expression regulation.

Further studies are necessary to fully identify the mobile elements in C. gattii and, especially, to completely assemble its genome(s). However, the presence of sRNAs as possible agents to control sequence mobilization creates a new basis for research on alternative pathways, AGO-like domains, and other mechanisms that promote non-canonical retrotransposon silencing in this medically relevant fungus.

\section{Methods \\ Retrotransposon mining}

Retrotransposon mining with LTR_Finder v1.0.6 [46], LTR_Harvest v1.5.7 [47], LTR_STRUC v1.1 [48], LTR_Seq [49], MGEScan v2 [50], and Transposon_PSI (http://transposonpsi.sourceforge.net) programs were used in C. gattii genomes with default parameters. The 17 C. gattii genomes (Table 2) from VGI, VGII, VGIII, and VGIV molecular types used in this work are available in the NCBI database .

New sequences were discovered by searching with the Blastn algorithm v2.2.31 (megablast) [51, 52] of known retrotransposons against genomes. Sequence confirmation by retrotransposon domain presence (gag, integrase, reverse transcriptase, RNase $H$, and aspartic protease) was evaluated using Blast Conserved Domains [53].

\section{Retrotransposon orthology between genomes}

Sequences that are putatively orthologous to R265 and WM276 retrotransposons were inferred by best bi-

Table 2 C. gattii strains used in retrotransposon mining

\begin{tabular}{lll}
\hline Molecular type & Strain & NCBI Reference number \\
\hline VGI & WM276 & GCA_000185945.1 \\
VGI & NT10 & GCA_000935105.1 \\
VGI & E566 & GCA_000875815.1 \\
VGI & EJB2 & GCA_000835745.1 \\
VGI & RU294 & GCA_000836355.1 \\
VGII & 99473 & GCA_000836455.1 \\
VGII & 2001935 & GCA_000835815.1 \\
VGII & CA1014 & GCA_000875795.1 \\
VGII & CBS7750 & GCA_000499585.1 \\
VGII & CBS10090 & GCA_000835765.1 \\
VGII & LA55 & GCA_000836315.1 \\
VGII & MMRL2647 & GCA_000875855.1 \\
VGII & R265 & GCA_000149475.3 \\
VGII & RAM5 & GCA_000836375.1 \\
VGIII & CA1280 & GCA_000836335.1 \\
VGIII & CA1873 & GCA_000855695.1 \\
VGIV & IND107 & GCA_000835755.1 \\
\hline
\end{tabular}


directional hits $(\mathrm{BBH}$ - with minimum 90\% query cover, $90 \%$ identity) in a Blastn search (megablast) with retrotransposon sequences against genomes. R265 retrotransposons distributed in all C. gattii strains (R265.3, R265.19, and R265.22) were tested for orthologous sequences in C. neoformans H99 (GCA_000149245.3) by the same methodology.

\section{Synteny evaluation}

Multiple genome alignment for synteny analysis of widely distributed R265.3, R265.19, and R265.22 orthologous sequences was performed in Mauve aligner v20150226 [54] with default parameters for all 17 C. gattii strains. Intrinsic synteny by orthologous retrotransposon localization inside VGI and VGII genotypes was analyzed on an independent multiple genome alignment of VGI and VGII strains. Syntenic blocks between genomes were represented by the same color.

\section{Phylogenetic inference}

Reverse trancriptase and RNase $\mathrm{H}$ domain sequences (nucleotide) from R265.19 and orthologous retrotransposons (17 C. gattii sequences and one C. neoformans H99 orthologue, as an outgroup) were separately aligned using MAFFT v7 [55] with default parameters for alignment strategy and adjusted sequence direction option. jModelTest v2.1.10 [56] was utilized to determine the best evolutionary model describing the data and Mr. Bayes v3.2.6 [57] was used for Bayesian analysis of phylogenetic inference. Computed trees were visualized with FigTree v1.4.2 (http://tree.bio.ed.ac.uk/software/figtree/) .

\section{C. gattii R265 RNA-Seq of small RNAs}

The C. gattii strain R265 was cultured in YPD media (2\% glucose; $2 \%$ peptone; $1 \%$ yeast extract) for $18 \mathrm{~h}$ at $200 \mathrm{rpm}$ and $30{ }^{\circ} \mathrm{C}$. Next, the cells were centrifuged $(5000 \times g-5 \mathrm{~min})$ and washed with $\mathrm{PBS}(\mathrm{NaCl}$ $137 \mathrm{mM} ; \mathrm{KCl} 2,7 \mathrm{mM} ; \mathrm{Na}_{2} \mathrm{HPO}_{4} 10 \mathrm{mM} ; \mathrm{KH}_{2} \mathrm{PO}_{4}$ 1,8 mM; pH 7,4). The cell pellet was resuspended in $20 \mathrm{~mL}$ of 1x YNB (Yeast Nitrogen Base) and diluted to $10^{7}$ cells $\left(\mathrm{OD}_{600}=1\right)$. For the zinc deprivation assay, the quantified cells were inoculated in $100 \mathrm{~mL}$ of YNB $1 \mathrm{x}+$ $10 \mu \mathrm{M}$ TPEN and kept for $18 \mathrm{~h}$ at $200 \mathrm{rpm}$. Afterwards, the cells were centrifuged (5000 g-5 min), frozen in liquid nitrogen, and placed in an ultrafreezer $\left(-80^{\circ} \mathrm{C}\right)$ for lyophilization.

RNA extraction was performed with Trizol $^{\circledR}$ (Invitrogen) reagent according to the manufacturer's protocol. Sample quantification was evaluated by fluorometric analysis with Qubit and Quant-It (Invitrogen), and samples were stored in RNA stable reagent (Biometrica). sRNAs were purified from total RNA samples, processed, and sequenced by Fasteris Life Sciences SA,
Plan-les-Ouates, Switzerland using Solexa technology on an Illumina Hi Seq 2000 platform.

\section{sRNAs prediction}

Reads from the zinc deprivation were filtered. Sequences from ribosomal RNAs (rRNAs), transporter RNAs (tRNAs), small nuclear RNAs (snRNAs), and small nucleolar RNAs (snoRNAs) present in the Rfam data bank [58] were excluded. Only sequences between 12 and 28 nucleotides were retained in the library. These sequences were aligned to Retrotransposon sequences using Bowtie2 [59]. Domains containing values of RPKM equal or larger than 500 RPKM were considered as possible sRNA targets.

\section{Expression evaluation}

The C. gattii R265 mRNA libraries of WT and ZAP1 depleted mutant (both grown in zinc deprivation conditions) used in this study were obtained from Schneider and colleagues [33].

RNA-Seq libraries (mRNAS and sRNAs) were aligned against the $C$. gattii R265 genome with the Tophat v2.1.1 program [60] unique alignment option (max-multihits $=1$ ). Mapped reads were visualized with IGV v2.3.78 [61] and quantified with Samtools v0.1.19 [62]. RPKM (reads Per kilobase per million) values were manually calculated and Log2 fold change was determined using the DESeq2 package of $\mathrm{R}$ [63]. Domain abundance inside retrotransposons was quantified by the division of raw read counts from each region by the sequence total read count. The statistical analysis of results was performed by ANOVA.

\section{Additional files}

Additional file 1: Retrotransposon sequence list from all 17 C. gattii strains. The retrotransposon list contains detailed descriptions of molecular type, C. gattii strain, NCBI reference for supercontig, sequence coordinates, LTR retrotransposon family and domains; as well as, the annotation of orthologous sequences in WM276 and R265 strains. The synteny analysis columns indicate presence ("YES") and absence ("NO") of synteny; and "NA" to not analyzed data. (XLS 118 kb)

Additional file 2: $C$. gattii multiple genome alignment. Multiple genome alignment of VGI, VGII, VGIII and VGIV molecular types with Mauve aligner. Syntenic blocks shared by genomes are represented with the same color and are connected by lines; red lines indicate chromosome or supercontig boundaries. (JPG $592 \mathrm{~kb}$ )

Additional file 3: VGI multiple genome alignment. Multiple genome alignment of strains from VGI molecular type with Mauve aligner. Syntenic blocks shared between genomes are represented with the same color and are connected by lines; red lines indicate chromosome or supercontig boundaries. (JPG $1033 \mathrm{~kb}$ )

Additional file 4: Retrotransposon-associated synteny breakpoints 1 in WM276. Figure shows a multiple genome alignment of VGI strains. Syntenic blocks shared by genomes are represented with the same color and are connected by lines; red lines indicate chromosome or supercontig boundaries. The location of associated retrotransposons WM276.1, WM276.2 WM276.3 and WM276.4 in chromosome A of WM276 strain is represented 
by the black rectangle. Microsynteny disruption by retrotransposon insertion can be observed as white spaces inside blocks. (JPG $308 \mathrm{~kb}$ )

Additional file 5: Retrotransposon-associated synteny breakpoints 2 in WM276. Figure shows a multiple genome alignment of VGI strains. Syntenic blocks shared by genomes are represented with the same color and are connected by lines; red lines indicate chromosome or supercontig boundaries. The location of associated retrotransposons WM276.26, WM276.75, WM276.27 and WM276.28 in chromosome E of WM276 strain is represented by the black rectangle. Microsynteny disruption by retrotransposon insertion can be observed as white spaces inside blocks. (JPG 304 kb)

Additional file 6: Retrotransposon-associated synteny breakpoints 3 in WM276. Figure shows a multiple genome alignment of VGI strains. Syntenic blocks shared by genomes are represented with the same color and are connected by lines; red lines indicate chromosome or supercontig boundaries. The location of associated retrotransposons WM276.44, WM276.46 and WM276.47 in chromosome H of WM276 strain is represented by the black rectangle. Microsynteny disruption by retrotransposon insertion can be observed as white spaces inside blocks. (JPG $328 \mathrm{~kb}$ )

Additional file 7: Retrotransposon-associated synteny breakpoints 4 in WM276. Figure shows a multiple genome alignment of VGI strains. Syntenic blocks shared by genomes are represented with the same color and are connected by lines; red lines indicate chromosome or supercontig boundaries. The location of associated retrotransposons WM276.52, WM276.53, WM276.54 and WM276.55 in chromosome J of WM276 strain is represented by the black rectangle. Microsynteny disruption by retrotransposon insertion can be observed as white spaces inside blocks. (JPG $356 \mathrm{~kb}$ )

Additional file 8: Retrotransposon-associated synteny breakpoints 5 in WM276. Figure shows a multiple genome alignment of VGI strains. Syntenic blocks shared by genomes are represented with the same color and are connected by lines; red lines indicate chromosome or supercontig boundaries. The location of associated retrotransposons WM276.72 and WM276.73 in chromosome N of WM276 strain is represented by the black rectangle. Microsynteny disruption by retrotransposon insertion can be observed as white spaces inside blocks. (JPG $329 \mathrm{~kb}$ )

Additional file 9: Retrotransposon-associated synteny breakpoints 1 in R265. Figure shows a multiple genome alignment of VGIl strains. Syntenic blocks shared by genomes are represented with the same color and are connected by lines; red lines indicate chromosome or supercontig boundaries. The location of associated retrotransposons R265.12 and R265.13 in supercontig 7 of R265 strain is represented by the black rectangle. Microsynteny disruption by retrotransposon insertion can be observed as white spaces inside blocks. (JPG $483 \mathrm{~kb}$ )

Additional file 10: Retrotransposon-associated synteny breakpoints 2 in R265. Figure shows a multiple genome alignment of VGll strains. Syntenic blocks shared by genomes are represented with the same color and are connected by lines; red lines indicate chromosome or supercontig boundaries. The location of associated retrotransposons R265.29, R265.30 and R265.31 in supercontig 25 of R265 strain is represented by the black rectangle. Microsynteny disruption by retrotransposon insertion can be observed as white spaces inside blocks. (JPG 436 kb)

Additional file 11: Retrotransposon-associated synteny breakpoints 3 in R265. Figure shows a multiple genome alignment of VGIl strains. Syntenic blocks shared by genomes are represented with the same color and are connected by lines; red lines indicate chromosome or supercontig boundaries. The location of associated retrotransposons R265.23 and R265.24 in supercontig 17 of R265 strain is represented by the black rectangle. Microsynteny disruption by retrotransposon insertion can be observed as white spaces inside blocks. (JPG $493 \mathrm{~kb}$ )

Additional file 12: Retrotransposon-associated synteny breakpoints 4 in R265. Figure shows a multiple genome alignment of VGIl strains. Syntenic blocks shared by genomes are represented with the same color and are connected by lines; red lines indicate chromosome or supercontig boundaries. The location of associated retrotransposons R265.26, R265.27, R265.34 and R265.28 in supercontig 21 of R265 strain is represented by the black rectangle. Microsynteny disruption by retrotransposon insertion can be observed as white spaces inside blocks. (JPG 508 kb)
Additional file 13: Retrotransposon-associated synteny breakpoints 5 in R265. Figure shows a multiple genome alignment of VGll strains. Syntenic blocks shared by genomes are represented with the same color and are connected by lines; red lines indicate chromosome or supercontig boundaries. The location of associated retrotransposons R265.38 and R265.20 in supercontig 13 of R265 strain is represented by the black rectangle. Microsynteny disruption by retrotransposon insertion can be observed as white spaces inside blocks. (JPG $487 \mathrm{~kb}$ )

Additional file 14: VGII multiple genome alignment. Multiple genome alignment of strains from VGII molecular type with Mauve aligner. Syntenic blocks shared by genomes are represented with the same color and are connected by lines; red lines indicate chromosome or supercontig boundaries. (JPG $2507 \mathrm{~kb}$ )

Additional file 15: Synteny analysis of R265.3, R265.19 and R265.22 orthologous sequences. Localization of orthologous retrotransposons of R265.3, R265.19, and R265.22 sequences are indicated by black lines in a multiple genome alignment with strains of VGI, VGII, VGIII, and VGIV molecular types. Orthologous sequences in each strain are named by the retrotransposon number. Syntenic blocks shared by genomes are represented with the same color and are connected by lines; red lines indicate chromosome or supercontig boundaries. (JPG $1397 \mathrm{~kb}$ )

Additional file 16: Raw read counts of mRNAs and sRNAs. Raw counts of mapped reads from mRNA and sRNA libraries to C. gattii R265 retrotransposons by the TopHat aligner. (XLSX $10 \mathrm{~kb}$ )

Additional file 17: Read counts values of retrotransposon sequences. Raw read counts distribution of retrotransposon sequences in WT and ZAP1 conditions. Distribution values are shown in a log scale. (JPG $27 \mathrm{~kb}$ )

Additional file 18: RPKM values of retrotransposon sequences. RPKM distribution of retrotransposon sequences in WT and ZAP1 conditions. Distribution values are shown in a log scale. (JPG $31 \mathrm{~kb}$ )

Additional file 19: Reads mapping profile of in full-length retrotransposons. Visualization of mapped reads profile from mRNA and sRNA libraries in full-length retrotransposons R265.5 and R265.7. Abbreviations: Long Terminal Repeats (LTR), Integrase (INT), Ribonuclease H (RNAse), Reverse Transcriptase (RT), Aspartyl Protease (PROT). (JPG 289 kb)

Additional file 20: Reads mapping profile of highly expressed retrotransposons. Visualization of mapped reads profile from mRNA and sRNA libraries in highly expressed sequences R265.19 and R265.31. Abbreviations: Long Terminal Repeats (LTR), Integrase (INT), Ribonuclease H (RNAse), Reverse Transcriptase (RT), Aspartyl Protease (PROT). (JPG 285 kb)

Additional file 21: Reads mapping profile of highly repressed retrotransposons. Visualization of mapped reads profile from mRNA and sRNA libraries in highly repressed sequences R265.36 and R265.37. Abbreviations: Long Terminal Repeats (LTR), Integrase (INT), Ribonuclease H (RNAse), Reverse Transcriptase (RT), Aspartyl Protease (PROT). (JPG 287 kb)

\section{Abbreviations}

dsRNA: Double stranded RNA; GAGs: Group-specific AntiGen; LTR: Long terminal repeats; $\mathrm{OD}_{600}$ : Optical density at $600 \mathrm{~nm}$; PBS: Phosphate buffered saline; RdRP: RNA dependent RNA polymerase; RISC: RNA-induced silencing complex; RNAi: Interfering RNA; ROS: Reactive oxygen species; RPKM: Reads Per Kilobase of tanscript per Million mapped reads; siRNA: Small interfering RNA; sRNA: Small RNA; TE: Transposable elements; TPEN: N,N,N', N'-tetrakis(2pyridinylmethyl)-1,2-ethanediamine; VG: Variety gattii; VLPs: Virus-Like Particles; WT: Wild type; YNB: Yeast nitrogen base; YPD: Yeast peptone dextrose

\section{Acknowledgment}

The authors would to thank Dr. Rodrigo Ligabue Braun for critical reading of the manuscript.

\section{Funding}

Financial support for this study was provided by Coordenação de Apoio de Pessoal de Nível Superior - CAPES (Process no. 23038.010041/2013-13) and Conselho Nacional de Desenvolvimento Científico e Tecnológico - CNPQ (Process no. 312408/2014-5). The funding agencies had no role in the design of the study and collection, analysis, and interpretation of data, as well as in writing the manuscript. 


\section{Availability of data and materials}

The data supporting the conclusions of this article will be available at the Sequence Read Archive (SRA) under codes SRR5209627, SRR5209175, and SRR5209145 in the NCBI GenBank repository as BioProject PRJNA368962.

\section{Authors' contributions}

Conceived and designed the experiments: PAGF RSAS FMS LK AS MHV CCS. Performed the experiments: PAGF RSAS FMS. Analyzed the data: PAGF CCS. Contributed reagents/materials/analysis tools: LK AS MHV CCS. Wrote the paper: PAGF CCS. All authors read and approved the final manuscript.

\section{Competing interests}

The authors declare that they have no competing interests.

\section{Consent for publication}

Not applicable.

Ethics approval and consent to participate

Nothing to declare.

\section{Publisher's Note}

Springer Nature remains neutral with regard to jurisdictional claims in published maps and institutional affiliations.

Received: 31 January 2017 Accepted: 6 April 2017

Published online: 12 April 2017

\section{References}

1. Chénais B, Caruso A, Hiard S, Casse N. The impact of transposable elements on eukaryotic genomes: from genome size increase to genetic adaptation to stressful environments. Gene. 2012;509:7-15.

2. Goodwin TJ, Poulter RT. The diversity of retrotransposons in the yeast Cryptococcus neoformans. Yeast. 2001;18:865-80.

3. Alzohairy AM, Gyulai G, Jansen RK, Bahieldin A. Transposable elements domesticated and neofunctionalized by eukaryotic genomes. Plasmid. 2013; 69:1-15.

4. Havecker ER, Gao X, Voytas DF. The diversity of LTR retrotransposons. Genome Biol. 2004:5:225

5. Nunes CC, Gowda M, Sailsbery J, Xue M, Chen F, Brown DE, Oh Y, Mitchell TK, Dean RA. Diverse and tissue-enriched small RNAs in the plant pathogenic fungus, Magnaporthe oryzae. BMC Genomics. 2011;12:288

6. Wang X, Hsueh YP, Li W, Floyd A, Skalsky R, Heitman J. Sex-induced silencing defends the genome of Cryptococcus neoformans via RNAi. Genes Dev. 2010;24:2566-82.

7. Moazed D. Small RNAs in transcriptional gene silencing and genome defence. Nature. 2009:457:413-20.

8. Janbon G, Maeng S, Yang DH, Ko YJ, Jung KW, Moyrand F, Floyd A, Heitman $J$, Bahn YS. Characterizing the role of RNA silencing components in Cryptococcus neoformans. Fungal Genet Biol. 2010;47:1070-80.

9. Dumesic PA, Natarajan P, Chen C, Drinnenberg IA, Schiller BJ, Thompson J, Moresco JJ, Yates JR, Bartel DP, Madhani HD. Stalled spliceosomes are a signal for RNAi-mediated genome defense. Cell. 2013;152:957-68.

10. Shi H, Djikeng A, Tschudi C, Ullu E. Argonaute protein in the early divergent eukaryote Trypanosoma brucei: control of small interfering RNA accumulation and retroposon transcript abundance. Mol Cell Biol. 2004; 24:420-7

11. Boesler B, Meier D, Förstner KU, Friedrich M, Hammann C, Sharma CM, Nellen W. Argonaute proteins affect siRNA levels and accumulation of a novel extrachromosomal DNA from the Dictyostelium retrotransposon DIRS1. J Biol Chem. 2014;289:35124-38.

12. Chaturvedi $V$, Chaturvedi S. Cryptococcus gattii: a resurgent fungal pathogen. Trends Microbiol. 2011;19:564-71.

13. Bielska E, May RC. What makes Cryptococcus gattii a pathogen? FEMS Yeast Res. 2016;16:fov106.

14. Okubo Y, Wakayama M, Ohno H, Yamamoto S, Tochigi N, Tanabe K, Kaneko Y, Yamagoe S, Umeyama T, Shinozaki M, et al. Histopathological study of murine pulmonary cryptococcosis induced by Cryptococcus gattii and Cryptococcus neoformans. Jpn J Infect Dis. 2013;66:216-21.

15. Farrer RA, Desjardins CA, Sakthikumar S, Gujja S, Saif S, Zeng Q, Chen Y, Voelz K, Heitman J, May RC, et al. Genome evolution and innovation across the four major lineages of Cryptococcus gattii. MBio. 2015;6:e00868-00815.
16. Feretzaki M, Billmyre RB, Clancey SA, Wang X, Heitman J. Gene network polymorphism illuminates loss and retention of novel RNAi silencing components in the Cryptococcus pathogenic species complex. PLoS Genet. 2016;12:e1005868.

17. Byrnes 3rd EJ, Li W, Lewit Y, Ma H, Voelz K, Ren P, Carter DA, Chaturvedi V, Bildfell RJ, May RC, et al. Emergence and pathogenicity of highly virulent Cryptococcus gattii genotypes in the northwest United States. PLoS Pathog. 2010;6:e1000850.

18. Billmyre RB, Calo S, Feretzaki M, Wang X, Heitman J. RNAi function, diversity, and loss in the fungal kingdom. Chromosome Res. 2013;21:561-72.

19. Servant $G$, Pennetier $C$, Lesage $P$. Remodeling yeast gene transcription by activating the Ty1 long terminal repeat retrotransposon under severe adenine deficiency. Mol Cell Biol. 2008;28:5543-54.

20. Lerat E. Identifying repeats and transposable elements in sequenced genomes: how to find your way through the dense forest of programs. Heredity. 2010;104:520-33.

21. Thon MR, Pan H, Diener S, Papalas J, Taro A, Mitchell TK, Dean RA. The role of transposable element clusters in genome evolution and loss of synteny in the rice blast fungus Magnaporthe oryzae. Genome Biol. 2006;7:R16.

22. Muszewska A, Hoffman-Sommer M, Grynberg M. LTR retrotransposons in fungi. PLoS One. 2011;6:e29425

23. Novikov A, Smyshlyaev G, Novikova O. Evolutionary history of LTR retrotransposon chromodomains in plants. Int J Plant Genomics. 2012;2012: 874743

24. D'Souza CA, Kronstad JW, Taylor G, Warren R, Yuen M, Hu G, Jung WH, Sham A Kidd SE, Tangen K, et al. Genome variation in Cryptococcus gattii, an emerging pathogen of immunocompetent hosts. MBio. 2011;2:e00342-00310.

25. Kimura M. A simple method for estimating evolutionary rates of base substitutions through comparative studies of nucleotide sequences. J Mol Evol. 1980:16:111-20

26. Servant G, Pinson B, Tchalikian-Cosson A, Coulpier F, Lemoine S, Pennetier C, Bridier-Nahmias A, Todeschini AL, Fayol H, Daignan-Fornier B, et al. Tye7 regulates yeast Ty 1 retrotransposon sense and antisense transcription in response to adenylic nucleotides stress. Nucleic Acids Res. 2012;40:5271-82.

27. Zaratiegui M. Influence of long terminal repeat retrotransposons in the genomes of fission yeasts. Biochem Soc Trans. 2013:41:1629-33.

28. VanHoute D, Maxwell PH. Extension of Saccharomyces paradoxus chronological lifespan by retrotransposons in certain media conditions is associated with changes in reactive oxygen species. Genetics. 2014;198:531-45.

29. Eide DJ. Homeostatic and adaptive responses to zinc deficiency in Saccharomyces cerevisiae. J Biol Chem. 2009;284:18565-9.

30. Wu CY, Roje S, Sandoval FJ, Bird AJ, Winge DR, Eide DJ. Repression of sulfate assimilation is an adaptive response of yeast to the oxidative stress of zinc deficiency. J Biol Chem. 2009;284:27544-56.

31. Wu CY, Bird AJ, Winge DR, Eide DJ. Regulation of the yeast TSA1 peroxiredoxin by ZAP1 is an adaptive response to the oxidative stress of zinc deficiency. J Biol Chem. 2007:282:2184-95.

32. Ballou ER, Wilson D. The roles of zinc and copper sensing in fungal pathogenesis. Curr Opin Microbiol. 2016;32:128-34

33. Schneider RO, Fogaça NS, Kmetzsch L, Schrank A, Vainstein MH, Staats CC. Zap1 regulates zinc homeostasis and modulates virulence in Cryptococcus gattii. PLoS One. 2012;7:e43773.

34. Zhao H, Eide DJ. Zap1p, a metalloregulatory protein involved in zincresponsive transcriptional regulation in Saccharomyces cerevisiae. Mol Cell Biol. 1997:17:5044-52.

35. Cavrak W, Lettner N, Jamge S, Kosarewicz A, Bayer LM, Mittelsten Scheid O. How a retrotransposon exploits the plant's heat stress response for its activation. PLoS Genet. 2014;10:e1004115.

36. Nakayashiki $\mathrm{H}$. The Trickster in the genome: contribution and control of transposable elements. Genes Cells. 2011;16:827-41.

37. Mourier T, Willerslev E. Large-scale transcriptome data reveals transcriptional activity of fission yeast LTR retrotransposons. BMC Genomics. 2010;11:167.

38. Domingues DS, Cruz GM, Metcalfe CJ, Nogueira FT, Vicentini R, Alves CS, Van Sluys MA. Analysis of plant LTR-retrotransposons at the fine-scale family level reveals individual molecular patterns. BMC Genomics. 2012;13:137.

39. Desjardins CA, Champion MD, Holder JW, Muszewska A, Goldberg J, Bailao AM, Brigido MM, Ferreira ME, Garcia AM, Grynberg M, et al. Comparative genomic analysis of human fungal pathogens causing paracoccidioidomycosis. PLoS Genet. 2011;7:e1002345.

40. Soares MA de Carvalho Araujo RA Marini MM, de Oliveira LM, de Lima LG, De Souza Alves V, Felipe MS, Brigido MM, de Almeida Soares CM, da Silveira JF, et 
al. Identification and characterization of expressed retrotransposons in the genome of the Paracoccidioides species complex. BMC Genomics. 2015;16:376,

41. Caza M, Kronstad JW. Shared and distinct mechanisms of iron acquisition by bacterial and fungal pathogens of humans. Front Cell Infect Microbiol. 2013;3:80.

42. Tristão $G B$, Assunção $L P$, Dos Santos $L P$, Borges $C L$, Silva-Bailão $M G$, Soares CM, Cavallaro G, Bailão AM. Predicting copper-, iron-, and zinc-binding proteins in pathogenic species of the Paracoccidioides genus. Front Microbiol. 2014;5:761.

43. Volpe TA, Kidner C, Hall IM, Teng G, Grewal SI, Martienssen RA. Regulation of heterochromatic silencing and histone $\mathrm{H} 3$ lysine-9 methylation by RNAi. Science. 2002;297:1833-7.

44. Lippman Z, May B, Yordan C, Singer T, Martienssen R. Distinct mechanisms determine transposon inheritance and methylation via small interfering RNA and histone modification. PLoS Biol. 2003;1:E67.

45. Saha A, Mitchell JA, Nishida Y, Hildreth JE, Ariberre JA, Gilbert WV, Garfinkel DJ. A trans-dominant form of Gag restricts Ty1 retrotransposition and mediates copy number control. J Virol. 2015;89:3922-38.

46. Xu Z, Wang H. LTR_FINDER: an efficient tool for the prediction of full-length LTR retrotransposons. Nucleic Acids Res. 2007;35:W265-8.

47. Ellinghaus D, Kurtz S, Willhoeft U. LTRharvest, an efficient and flexible software for de novo detection of LTR retrotransposons. BMC Bioinformatics. 2008:9:18.

48. McCarthy EM, MCDonald JF. LTR_STRUC: a novel search and identification program for LTR retrotransposons. Bioinformatics. 2003;19:362-7.

49. Kalyanaraman A, Aluru S. Efficient algorithms and software for detection of full-length LTR retrotransposons. J Bioinform Comput Biol. 2006;4:197-216.

50. Lee H, Lee M, Mohammed Ismail W, Rho M, Fox GC, Oh S, Tang H. MGEScan: a Galaxy-based system for identifying retrotransposons in genomes. Bioinformatics. 2016;32:2502-4.

51. Altschul SF, Gish W, Miller W, Myers EW, Lipman DJ. Basic local alignment search tool. J Mol Biol. 1990;215:403-10.

52. Morgulis A, Coulouris G, Raytselis Y, Madden TL, Agarwala R, Schäffer AA. Database indexing for production MegaBLAST searches. Bioinformatics. 2008;24:1757-64.

53. Marchler-Bauer A, Derbyshire MK, Gonzales NR, Lu S, Chitsaz F, Geer LY, Geer RC, He J, Gwadz M, Hurwitz DI, et al. CDD: NCBI's conserved domain database. Nucleic Acids Res. 2015:43:D222-6.

54. Darling AC, Mau B, Blattner FR, Perna NT. Mauve: multiple alignment of conserved genomic sequence with rearrangements. Genome Res. 2004;14 1394-403.

55. Katoh K, Standley DM. MAFFT multiple sequence alignment software version 7: improvements in performance and usability. Mol Biol Evol. 2013; 30:772-80.

56. Darriba D, Taboada GL, Doallo R, Posada D. jModelTest 2: more models, new heuristics and parallel computing. Nat Methods. 2012;9:772.

57. Huelsenbeck JP, Ronquist F. MRBAYES: Bayesian inference of phylogenetic trees. Bioinformatics. 2001;17:754-5.

58. Burge SW, Daub J, Eberhardt R, Tate J, Barquist L, Nawrocki EP, Eddy SR Gardner PP, Bateman A. Rfam 11.0: 10 years of RNA families. Nucleic Acids Res. 2013:41:D226-32.

59. Langmead B, Salzberg SL. Fast gapped-read alignment with Bowtie 2. Nat. Methods. 2012;9:357-59.

60. Kim D, Pertea G, Trapnell C, Pimentel H, Kelley R, Salzberg SL. TopHat2: accurate alignment of transcriptomes in the presence of insertions, deletions and gene fusions. Genome Biol. 2013;14:R36.

61. Thorvaldsdóttir H, Robinson JT, Mesirov JP. Integrative Genomics Viewer (IGV): high-performance genomics data visualization and exploration. Brief Bioinform. 2013;14:178-92.

62. Li H, Handsaker B, Wysoker A, Fennell T, Ruan J, Homer N, Marth G, Abecasis G, Durbin R, Subgroup GPDP. The Sequence Alignment/Map format and SAMtools. Bioinformatics. 2009:25:2078-9.

63. Love Ml, Huber W, Anders S. Moderated estimation of fold change and dispersion for RNA-seq data with DESeq2. Genome Biol. 2014;15:550.

\section{Submit your next manuscript to BioMed Central and we will help you at every step:}

- We accept pre-submission inquiries

- Our selector tool helps you to find the most relevant journal

- We provide round the clock customer support

- Convenient online submission

- Thorough peer review

- Inclusion in PubMed and all major indexing services

- Maximum visibility for your research

Submit your manuscript at www.biomedcentral.com/submit

) Biomed Central 